\title{
LA NECESIDAD DE FORTALECER \\ LA PROTECCIÓN DE LA PROPIEDAD \\ INTELECTUAL EN LOS TEJIDOS WAYÚU \\ DEBIDO A LAS NUEVAS DINÁMICAS \\ DE PRODUCCIÓN EN LA COMUNIDAD
}

\author{
MAYERLIN MEJÍA PAREJA*
}

\section{RESUMEN}

Las nuevas dinámicas de producción, acceso a materias primas y comercialización de los tejidos wayúu exigen la aplicación de normas sui generis de protección intelectual colectiva, para garantizar los derechos de la comunidad a través de la venta de sus artesanías. Los mecanismos legales vigentes asociados a la protección de la propiedad intelectual no contemplan la creación colectiva ni consideran las relaciones de las comunidades indígenas con sus creaciones, su valor cultural y simbólico, el cual se desconoce en medio de los nuevos procesos de producción y comercialización. Esta situación obliga a analizar con detalle el tema de la propiedad intelectual para esta clase de productos.

Palabras clave: Propiedad Intelectual; Tejidos wayúu; Procesos Productivos; Comercialización; Derechos; Valor Cultural.

\section{ABSTRACT}

The new dynamics of production, access to raw materials and commercialization of Wayuu fabrics require the application of sui generis norms of collective intellectual protection, to guarantee the rights of the community through the sale of their handicrafts. The current legal mechanisms associated with the protection of intellectual property do not contemplate collective creation nor do they consider

* Ingeniera Industrial de la Universidad de La Guajira, Especialista en Gerencia de Recursos Humanos de la Universidad del Norte, Magíster en Derecho Privado, Persona y Sociedad con énfasis en Propiedad Intelectual de la Universidad Externado de Colombia. Rioacha (Colombia). Correo-e: mayemejia31@gmail.com. Fecha de recepción: 8 de abril de 2020. Fecha de aceptación: 30 de julio de 2020. Para citar el artículo: Mejía Pareja, Mayerlin. "La necesidad de fortalecer la protección de la propiedad intelectual en los tejidos wayúu debido a las nuevas dinámicas de producción en la comunidad", en Revista de la propiedad inmaterial n. ${ }^{\circ} 30$, Universidad Externado de Colombia, julio 2020-diciembre 2020, pp. 229-246. Dor: https://doi.org/10.18601/16571959.n30.09 
the relations of indigenous communities with their creations, their cultural and symbolic value, which is unknown in the midst of the new production and commercialization processes. This situation requires a detailed analysis of the issue of intellectual property for this class of products.

Keywords: Intellectual Property; Wayuu Fabrics; Production Processes; Commercialization; Rights; Cultural Value.

\section{INTRODUCCIÓN}

El Wayúu es un pueblo indígena establecido principalmente en el departamento de La Guajira, en el norte de Colombia, y han logrado mantener su cultura y sus tradiciones a través del desarrollo y trasformación de sus actividades productivas (Vázquez, 2006). Sin embargo, un elemento clave que sigue haciendo parte de la comunidad, que orienta las dinámicas sociales, culturales y económicas de sus integrantes, y que además establece profundas relaciones entre las generaciones y las familias es el tejido y la artesanía.

En particular, como lo explica Sánchez ${ }^{1}$, el tejido wayúu ha sido clave no solo como un medio de sobrevivencia sino también con un elemento que representa una serie de procesos culturales, los cuales determinan el sentido de pertenencia, las relaciones que establecen las personas con el territorio, el acceso a los recursos y la forma en que se utilizan los bienes producidos por la naturaleza para garantizar el desarrollo de la comunidad.

Sin embargo, como todo proceso productivo en la actualidad, el tejido Wayúu ha tenido que adaptarse a las nuevas exigencias y dinámicas del mercado ${ }^{2}$. Por ejemplo, siguiendo la argumentación planteada por Sánchez ${ }^{3}$ anteriormente la comunidad usaba sus tejidos -dentro de los que se destacan las mantas, vestidos, sombreros, mochilas y bolsos- como medio de intercambio con otras comunidades a través del trueque.

No obstante, debido a las trasformaciones geográficas que han existido en el territorio, se ha generado una separación progresiva de las tribus indígenas y de las comunidades, a causa del desarrollo de proyectos de infraestructura y de nuevas visiones que conciben al territorio más como un medio de modernización y competitividad económica que como un legado ancestral y espiritual ${ }^{4}$. En este contexto, los wayúu han tenido que orientar el desarrollo de nuevas estrategias de

1 Sánchez, B. "Espiritualidad Wayuu en relación con la muerte", en Revista de Literatura Hispanoamericana, 2016. 2(4), pp. 22-54.

2 Polo, N. "A gaze to Wayu's Culture, Base of his Normative System", en Paripex -Indian Journal of Research, 4(250), 2015, pp. 72-75..

3 SÁnchez, B. "Una aproximación al arte wayuu en; el lenguaje simbólico del mito", en Revista de Literatura Hispanoamericana, 2003, 2(2), pp. 33- 44.

4 Polo, N. La cultura y el sistema de normas Wayuu. Nuevo Derecho, 11(17), $65-$ 78. 2015. 
LA NECESIDAD DE FORTALECER LA PROTECCIÓN DE LA PROPIEDAD INTELECTUAL EN LOS

TEJIDOS WAYÚU DEBIDO A LAS NUEVAS DINÁMICAS DE PRODUCCIÓN EN LA COMUNIDAD

producción y distribución de sus tejidos que sean más coherentes con la demanda y las nuevas técnicas de mercadeo 5 .

El desarrollo de nuevas estrategias y medios de producción en la comunidad se ha generado también debido a una transformación creciente en la demanda de este tipo de artesanías, ya que últimamente han sido valoradas en medio de las políticas de recuperación de los valores indígenas en Colombia ${ }^{6}$. Nuevamente, Sánchez ${ }^{7}$ indica que actualmente los tejidos wayúu se han comenzado a comercializar incluso en el mercado internacional, debido no solo a su valor cultural sino también a las propiedades físicas y estéticas de cada uno de productos.

Se evidencia un problema en el cual no se ha logrado articular el hecho de que las artesanías wayúu sean un símbolo de conservación cultural y al mismo tiempo un negocio en el comercio exterior ${ }^{8}$. Como lo explica Manquillo ${ }^{9}$, la articulación dependería del desarrollo de estrategias efectivas por medio de las cuales se apoyara el desarrollo de la comunidad, la conservación y difusión de su cultura, mientras que al mismo tiempo se generan nuevas oportunidades de crecimiento económico y productividad para la comunidad y para el país a través de los procesos de comercialización en el exterior ${ }^{10}$.

En efecto, los tejidos wayúu han fascinado varios segmentos del mercado porque ayudan a promover los valores culturales y la sabiduría de una de las tribus indígenas más antiguas en Latinoamérica, y también por la viveza de sus colores, la complejidad de los diseños y de las técnicas que son implementadas para su elaboración, como lo esbozan Polo y Ojeda ${ }^{11}$. En tal sentido, los tejidos wayúu representan elementos de la naturaleza, como las estrellas, los animales y las plantas, además de todo un conjunto de símbolos ancestrales que hacen parte de la vida en comunidad, los cuales deben ser protegidos en el contexto de la Propiedad Intelectual, ya que se generaría un escenario de oportunidades para los artesanos y las comunidades que viven de ello en el país ${ }^{12}$.

En conjunto, todos estos valores y atributos de los tejidos han sido explotados con fines asociados a la comercialización. En este proceso se incluye la asesoría de

5 Hostein, N. El pueblo wayuu de La Guajira colombo-venezolana: un panorama de su cultura. Cuadernos de Antropología, 20. 2010.

6 Saler, B. "Los wayú (guajiro)", en Los aborígenes de Venezuela, Caracas, Fundación La Salle y Monte Ávila. 1987.

7 Sánchez, B. 2016, Op. cit.

8 Apshana, V. En: Harker, S. Wayuu peuple du désert colombien. Bogotá, Villegas Editores. 1998.

9 Manquillo, N. 2. "La vulnerabilidad del sector artesanal colombiano en el ámbito nacional e internacional como 'negocio de conocimiento tradicional. Estudio de caso: la denominación de origen artesanal 'Guacamayas' en el Acuerdo Comercial entre Colombia, Perú y la Unión E”. Revista La Propiedad Inmaterial. 28 (dic. 2019), pp. 167-209.

10 Alarcón, J. The Wayuu society, between chimera and reality. Gazeta de Antropología, 2006, p. 22, artículo 21.

11 Polo, N. y Ojeda, G. "Mirada a la cultura Wayuu, base de su sistema normativo", en Verbum, 2014, 9(9), pp. 109-117.

12 Buitrago, C. Artesanias y propiedad intelectual. ¿Cuál es la situación legal de las artesanias en Colombia? Universidad de Manizales, 2015. 
diseñadores que no necesariamente conocen los valores y las simbologías de las comunidades, con la finalidad de hacer más populares y comerciales los productos, según manifiestan Aguilar y Márquez ${ }^{13}$. El problema central, por tanto, radica en que a partir del desarrollo de nuevas estrategias de producción para favorecer la venta de los tejidos y de una estrategia que responde a políticas nacionales de recuperación de los valores tradicionales de las comunidades indígenas, se puede estar generando como resultado una continua y sistemática violación de la propiedad intelectual de la comunidad Wayúu ${ }^{14}$.

A este respecto, la Organización Mundial de Propiedad intelectual indica que "La propiedad intelectual se asocia con esquemas de protección a las creaciones e invenciones producto de la mente humana, dentro de las que se destacan las obras literarias y artísticas, símbolos, nombres e imágenes utilizados en el comercio" ${ }^{15}$. Sin embargo, los esquemas de protección intelectual a nivel mundial han sido débiles en lo que se refiere a la propiedad colectiva, pues se han orientado centralmente a la creación concreta de individuos, pero no de comunidades, como lo establecen Vargas y Lascarro ${ }^{16}$.

Se puede reconocer que en la actualidad elementos como el desarrollo de la tecnología, los medios, la información y la cultura han generado como resultado avances importantes en el sistema jurídico de la Propiedad Intelectual ${ }^{17}$. En efecto, como lo explican Benavides y Forero ${ }^{18}$ se observa que la propiedad intelectual ha venido experimentando una profunda evolución que le permita ajustarse a los nuevos retos en materia de protección para las nuevas creaciones intelectuales.

Como lo explica Silberleib ${ }^{19}$, el desarrollo tecnológico también ha generado nuevas opciones para favorecer la creación y la producción, lo cual va de la mano con la necesidad de satisfacer las necesidades cada vez más exigentes de los consumidores y del mercado en general. De esta manera, teniendo en cuenta las dinámicas que orientan el desarrollo de procesos y productos de innovación, además de la globalización, apertura económica, desarrollo tecnológico y crecimiento continuo en la producción de activos inmateriales, cada Estado debe contar con garantías

13 Aguilar, I. y Márquez, P. El arte del tejido entre los hombres de la etnia wayúu de La Guajira colombo-venezolana, Colombia, Fundación Universidad de América, 2016.

14 Sánchez, $O p$, cit., p. 44.

15 Organización Mundial de la Propiedad Intelectual. [online] ¿Qué es la Propiedad Intelectual?, 2017. Disponible en: http://www.wipo.int/edocs/pubdocs/es/intproperty/450/ wipo_pub_450.pdf

16 Vargas, S., y Lascarro, A. "Derecho a la autodeterminación de los pueblos indígenas en el ordenamiento jurídico colombiano”, en Revista de Estudios Sociales, 2015, (53), pp. 65-76.

17 Acea, Y. (2016). "El sistema de propiedad intelectual y la protección de los conocimientos tradicionales. Apuntes necesarios", en Alegatos, 12(3), pp. 25-55.

18 Benavides, G., Óscar, A. \& Forero, P. (2002). "Crecimiento endógeno: conocimiento y patentes”, en Revista de Economía Institucional, 4(6), pp. 109-131.

19 Silberleib, L. "El derecho, la propiedad intelectual y el entorno digital", en Información, Cultura Y Sociedad, 1, (4), 2011, pp. 34-66. 
que les permitan proteger sus ideas y sus creaciones, a través de un ajuste adecuado de las leyes asociadas a la propiedad intelectual y al derecho de autor ${ }^{20}$.

Sin embargo, este avance no ha sido evidente en el desarrollo de esquemas de protección para la propiedad colectiva en lo que se refiere a la creación de artesanías. Se reconoce que las leyes y la jurisprudencia asociadas a la propiedad intelectual dependen de las necesidades de cada Estado, de las particularidades a través de las cuales se contempla la relevancia de los derechos de autor para garantizar el bienestar social y como motor relevante en la competitividad y desarrollo económico ${ }^{21}$.

EL tema de la propiedad intelectual es mucho más complejo aun cuando se hace referencia a los tejidos wayúu, que son el resultado de una serie de valores tradicionales y culturales únicos, que no son muchas veces compatibles con las dinámicas del mercado y con los esquemas clásicos de producción ${ }^{22}$. Por tanto, el objetivo central del presente artículo es analizar la posibilidad de promover el desarrollo de un sistema de propiedad intelectual orientado a las expresiones tradicionales culturales de los pueblos indígenas, que favorezca la protección de los conocimientos tradicionales en tejeduría del pueblo Wayúu.

\section{EL SENTIDO Y EL SIGNIFICADO DEL TEJIDO PARA LA COMUNIDAD WAYÚU}

El arte de tejer dentro de la comunidad es algo que se transmite de generación en generación, principalmente de madres a hijas. Hay que tener en cuenta, siguiendo las palabras de Barrera, Quiñonez y Jacanamijoy ${ }^{23}$, que los tejidos no solo están presentes en artesanías y en prendas de vestir, sino también en piezas que son de gran utilidad en la comunidad, como las redes de pesca y los chinchorros. De esta manera, el tejido se vincula a una manera particular de usar y aprovechar los recursos naturales con la finalidad de mejorar el bienestar y la calidad de vida al interior de la tribu ${ }^{24}$. De acuerdo con Pérez $^{25}$ :

Las artesanías son símbolos emblemáticos de una nación, su connotación sociocultural se arraiga a los auténticos conocimientos tradicionales y costumbristas regionales que

20 Rangel, H. La observancia de los derechos de propiedad intelectual, Organización Mundial de la propiedad Intelectual, 2015.

21 Tejedor, M.; Gil, F. y Tejedor, P. "Derechos de propiedad intelectual (DPI) y crecimiento económico: una revisión". Rev. Fac. Cienc. Econ, 16(1), 2016, pp. 225-243.

22 Sempere, A. Propiedad intelectual conceptos básicos, España, Universidad de Alicante, 2015.

23 Barrera, G., Quiñonez, A., y Jacanamijoy, J. "Riesgos y tensiones de las marcas colectivas y denominaciones de origen de las creaciones colectivas artesanales indígenas", en Apuntes. 2009., 27(1), 36-51.

24 López, A. La experiencia de Panamá respecto a la protección de las artesanías y su relación con la propiedad intelectual. Disponible en: http://www.impi.gob.mx/work/sites/ IMPI/resources/LocalContent/2383/1/ARESIO.pdf (20 de septiembre del 2011).

25 Pérez, J. "La artesanía en el Derecho", en Vniversitas 125, 2012, pp. 287-318. 
generan derechos intangibles, los cuales merecen ser protegidos legalmente, con el fin de facilitar su desarrollo como un verdadero sector económico.

En este sentido, como emblema cultural, las artesanías merecen contar con una protección especial, no solo porque tienen una participación en el mercado, sino además porque hacen parte de la representación cultural del país ${ }^{26}$. Al constituir el patrimonio público de la nación no deben ser excluidos del desarrollo de mecanismos efectivos de protección, sino que, por el contrario, deben contar con mejores medidas de amparo, sobre todo teniendo en cuenta que la producción de las artesanías se relaciona con un conjunto de cosmovisiones y prácticas ancestrales ${ }^{27}$.

Otro rasgo importante es que el tejido está relacionado con el linaje y prestigio de las familias. Esto quiere decir que entre mayores habilidades muestren las mujeres con sus tejidos, más admiración y respeto tendrán dentro de la comunidad ${ }^{28}$. El tejido wayúu es una práctica ancestral que es cuidada por el clan, de tal manera que los conocimientos y las técnicas no se comparten con personas que no hagan parte de la comunidad, como lo indican Aguilar y Márquez ${ }^{29}$. De esta manera, se puede observar que el tejido representa también un rasgo cultural e identitario de los wayúu, que devela una manera única de crear y de producir a partir de un conjunto de simbologías en torno al territorio, los recursos naturales y los conocimientos ancestrales.

No se sabe con exactitud, desde una perspectiva histórica, en qué momento la comunidad empezó a utilizar el tejido como medio de supervivencia y de afirmación de una identidad particular. Generalmente se dicen cosas como que la comunidad nació tejiendo, y que es una actividad que ha estado presente en cada generación, totalmente vinculada a sus costumbres y tradiciones más antiguas, indican nuevamente Barrera, Quiñonez y Jacanamijoy ${ }^{30}$. La única versión concreta que existe sobre el origen del tejido wayúu responde a una leyenda, la cual es preciso considerar con el fin de entender mejor cuál es el verdadero sentido y significado de esta actividad para la comunidad.

La leyenda habla de una araña conocida como Walekerü, que bajo la luz de la luna tejía mantas y mochilas. En una de esas noches iluminadas por la luna una niña se le acercó a la araña para contemplar su maravillosa habilidad en el tejido. La araña se sintió halagada y se ofreció para enseñarle a la niña cómo tejer. Así, a lo largo de varias noches la niña estuvo con la araña tejiendo, hasta que alcanzó la habilidad de su maestra. Cuando la niña llegó a su edad adulta la araña desapareció por siempre, pero dejaría un legado enorme para la comunidad el arte del tejido.

26 Díaz, A. América Latina y el Caribe: la propiedad intelectual después de los tratados de libre comercio, CEPAL, 2008, Santiago de Chile.

27 Rubio, A et ál. Guía de la artesanía y el derecho de autor. Coautoría de Artesanías de Colombia y la Dirección de Derecho de Autor. 2010.

28 URIBE, M. La transformación de la Propiedad Intelectual, Ediciones Doctrina y Ley, 2005, Bogotá D.C.

29 Aguilar, I. y Márquez, P. Op. cit.

30 Barrera, G.; Quiñonez, A. y Jacanamijoy, J. Op. cit. 
LA NECESIDAD DE FORTALECER LA PROTECCIÓN DE LA PROPIEDAD INTELECTUAL EN LOS

TEJIDOS WAYÚU DEBIDO A LAS NUEVAS DINÁMICAS DE PRODUCCIÓN EN LA COMUNIDAD

De acuerdo con esta leyenda, se puede apreciar que el tejido se encuentra inscrito dentro de la cosmovisión particular de las wayúu, de las relaciones que establecen los miembros de la comunidad con la naturaleza y con los demás seres vivos. En efecto, como lo señala Chávez ${ }^{31}$ cada uno de los símbolos que se incluyen en los tejidos representa figuras importantes para el pueblo Wayúu. Se tejen figuras abstractas, llamadas kanaas, que hacen alusión a la fauna y a la flora autóctona de la región. Incluso los tejidos que no tienen figuras como tal sino un conjunto de líneas, representan, de acuerdo con su dirección y el punto en el que se cruzan los caminos del pueblo, las varas de los ranchos, las huellas que dejan los caballos en la tierra, las vísceras de las vacas y los caparazones del morrocoy. A este respecto, la empresa Artesanías de Colombia precisa:

Los motivos tradicionales de los kanaas son la expresión más auténtica del modo como los wayúu interpretan y abstraen elementos de su mundo material, de su vida cotidiana, para crear figuras estilizadas de gran simbolismo, entre ellos se destacan los genitales de asnos, los caparazones de tortugas y las constelaciones de estrellas, entre otros. ${ }^{32}$

El hecho de que hoy en día elementos artesanales como las mochilas wayúu estén en auge en el ámbito de la moda y hayan ganado una importante participación en el mercado, ha sido beneficioso para la comunidad, en la medida en que ahora tienen un mayor reconocimiento por parte de la sociedad en general, y, así mismo, han logrado mejorar su capacidad de producción para enfrentar la creciente demanda; sin embargo y en el mismo orden de ideas, esta situación también ha generado efectos adversos y ha resultado ser bastante perjudicial, ya que el proceso de expansión comercial no ha sido regulado a través de normas específicas de propiedad intelectual colectiva de manera efectiva.

Lo anterior ha generado como resultado que existan cada vez más marcas y empresas que se dedican a comercializar los tejidos wayúu, en la mayoría de los casos sin el debido permiso de la comunidad, adquiriéndolas además a precios muy bajos y vendiéndolas por valores exorbitantes, como lo indican Barrera, Quiñonez y Jacanamijoy ${ }^{33}$. Adicionalmente, otro sector se dedica a producir imitaciones casi idénticas de manera industrial en entornos urbanos, lo cual va en contravía del valor cultural y patrimonial que significa la artesanía ancestral producida en el seno mismo de la comunidad por sus mujeres.

De acuerdo con el Plan Salvaguarda de la Comunidad Wayúu ${ }^{34}$, la realidad es que en Colombia hay muchas empresas que comercializan las mochilas en el

31 Chávez, C. Cultura y globalización: la seguridad societal de la comunidad indigena wayuu y la comercialización de los tejidos tradicionales, Universidad Colegio Mayor de Nuestra Señora del Rosario, 2017.

32 Artesanías de Colombia. Memoria de oficio tejeduría del pueblo indígena wayúu. [En linea]. 2014. Disponible en: https://www.artesaniasdecolombia.com.co/PortalAc/c_noticias/ la-mochila-wayu-parte-de-la-tradicion-de-colombia_5070

33 Barrera, G.; Quiñonez, A. y Jacanamijoy, J. Op. cit.

34 Comunidad Wayúu. Plan salvaguarda del pueblo Wayúu. [En línea].2014. Disponible 
mercado internacional, bajo la premisa de que estas son de fabricación artesanal wayúu, cuando en realidad no lo son. Ante lo cual, la principal recomendación de la comunidad a quienes quieran hacerse con una auténtica mochila de un tejido de este tipo, lo hagan sin intermediarios, adquiriéndole el producto directamente a los miembros de la misma población wayúu.

En síntesis, es preciso avanzar en el fortalecimiento de los esquemas de producción teniendo en cuenta que hoy en día la comunidad indígena no produce solamente los tejidos para ellos mismos, ni como medio de trueque con otras comunidades ancestrales, sino que estos productos han entrado en una dinámica compleja de comercialización en el exterior, sujeta a la voluntad de quienes inflan los precios sin retribuir lo justo a los artesanos y permitiendo la proliferación de copias e imitaciones. Por tanto, antes de entrar a analizar las orientaciones específicas para el desarrollo de medidas de protección intelectual colectiva para la comunidad, es preciso evaluar de qué manera se han establecido las transformaciones en los procesos de producción del tejido wayúu.

\section{TRANSFORMACIONES EN LOS MEDIOS Y PROCESOS \\ DE PRODUCCIÓN DEL TEJIDO WAYÚU}

A lo largo de los últimos años han venido cambiando los procesos por medio de los cuales se transforman los recursos naturales y las formas en las cuales se adquieren las materias primas para los tejidos wayúu. En la época precolombina, todo se basaba en el algodón. En la zona de La Guajira baja los cultivos de algodón eran muy comunes, y principalmente eran las abuelas las que tenían y cuidaban las plantaciones. El proceso posterior era convertir al algodón en hilaza y en hilo. Las comunidades wayúu que no cultivaban el algodón lo obtenían a través de trueques; nuevamente el documento de Artesanías de Colombia indica que:

Los Wayuu hilan el algodón silvestre, el magüey en mecha, el aipis y otras fibras naturales propias de la región. Tuercen cintas de cuerdo de chivo o de res para formar hilos, cuerdas y cordeles. Retuercen el algodón y la lana industrial para los tejidos finos, además de los hilos gastados que reutilizan en los tejidos "de segunda". Ya sea "en pierna", o con la ayuda del huso, los Wayuu tuercen y retuercen hilos en forma de "S" o en "Z"35.

Sin embargo, los procesos de desarrollo de infraestructura en la región limitaron progresivamente las plantaciones de algodón, de tal manera que los wayúu tuvieron que utilizar el hilo ya procesado como materia prima, el cual se obtenía no a partir de procesos de siembra desarrollados por las abuelas, sino a través de un proceso

en: https://siic.mininterior.gov.co/sites/default/files/pueblo_wayuu_norte_albania_-_diagnostico_comunitario.pdf

35 Artesanías de Colombia. Op. cit., p. 1. 
natural de compra en el mercado. Con la llegada del hilo también llegaron los colores, pues con el algodón todos los tejidos eran blancos. Por tanto, el uso de hilo generó nuevas posibilidades de creación para los indígenas, ya que asociaban los colores con sueños, símbolos o elementos de la naturaleza.

Posteriormente, a comienzos del siglo xxI, las comunidades indígenas entraron a participar en procesos de capacitación con el Sena y con Artesanías de Colombia para aprender mejores técnicas de producción, con la finalidad de mejorar la eficiencia en el proceso. Las mujeres ya no solo sabían tejer, sino también hacer un diseño y perfeccionar los productos, para de esta manera mejorar su impacto comercial.

En medio de este proceso de apertura, no solo se han introducido nuevas técnicas, sino que también se han generado variaciones en los diseños dependiendo de las necesidades y de las preferencias de los clientes. A continuación, se explican algunos de los elementos clave en el proceso que ha orientado una progresiva trasformación de la producción de los tejidos wayúu:

- Principalmente se utilizó como materia prima el algodón, las fibras vegetales y el fique.

- En medio de los procesos de colonización se introdujo como elemento clave en los tejidos la lana de oveja y la crin de caballo.

- En el periodo de colonización el algodón se usó solamente para hacer los tejidos de las castas que tenían mayor poder adquisitivo en las comunidades.

- Por otro lado, las castas de más baja condición económica tejían con trupillo y maguey.

- Hoy en día, con la finalidad de atender a la demanda que se ha generado, las indígenas trabajan principalmente con acrílico en sus tejidos, ya que es el material más económico ${ }^{36}$.

El problema es que las simbologías de los wayúu y las dinámicas que han venido afectando y trasformando sus procesos de producción no se dan a conocer en los procesos de comercialización, y tampoco se protegen mediante mecanismos efectivos de propiedad intelectual colectiva ${ }^{37}$. Por tanto, a continuación, se presentan orientaciones para promover el desarrollo de mejores esquemas que garanticen los derechos de autor de la comunidad wayúu, comprendida en este caso como una población con una cosmovisión y una cultura particular desde la cual se orienta la creación artística de los tejidos.

\section{ORIENTACIONES PARA EL FORTALECIMIENTO}

\section{DE LOS PROCESOS DE PROPIEDAD INTELECTUAL COLECTIVA}

La principal problemática que se ha venido evidenciando es que los tejidos wayúu han entrado en los últimos años en nuevas lógicas de venta, comercialización y

36 Guerra, W. “Los conflictos interfamiliares Wayúu”, Fronesis, 13(1), 2006, pp. 40-56.

37 Mejía, P. Situación sociolingüistica del Wayuunaiki. (Tesis de maestría). Universidad Nacional de Colombia, Bogotá, Colombia, 2011. 
distribución que van más allá de los procesos ancestrales de intercambio como el trueque, lo cual ha generado importantes afectaciones en los procesos naturales de producción de la comunidad. En medio de estas trasformaciones, el Estado no ha logrado implementar mecanismos efectivos de protección de la propiedad intelectual colectiva para la comunidad, pues principalmente se ha asumido una concepción según la cual las obras de los pueblos indígenas son patrimonio nacional, y por lo tanto pertenecen al Estado ${ }^{38}$.

En medio de este desconocimiento de la necesidad de proteger los derechos de la comunidad sobre sus creaciones artesanales y artísticas, hoy en día son múltiples los casos de empresas que buscan en los tejidos wayúu nuevas posibilidades para aplicar en distintos tipos de productos, como mochilas, vestuario, muebles y objetos para el hogar, con la finalidad ofrecer nuevos elementos simbólicos y estéticos que atraigan la atención del consumidor ${ }^{39}$. En palabras de Barrera, Quiñonez y Jacanamijoy ${ }^{40}$, se está utilizando la excusa de rescatar los valores y las expresiones culturales y artísticas de los pueblos indígenas en Colombia con la verdadera finalidad de aumentar las ventas.

En este sentido, los mecanismos de propiedad intelectual que se han aplicado sobre las mochilas wayúu están ligados a los procesos de internacionalización de las empresas que las están comercializando en el exterior, de tal manera que dichos mecanismos están protegiendo es a las compañías, pero no a la comunidad wayúu; continúan los autores:

A nivel general, se considera que estos mecanismos de implementación de derechos de propiedad intelectual sobre artesanías indígenas tienen una estructura que pueden propiciar procesos de dependencia institucional, cultural, económica, jurídica y política al vincular a los diversos pueblos indígenas artesanales con marcas colectivas y denominaciones de origen ${ }^{41}$.

El principal problema que limita la aplicación concreta de esquemas de propiedad intelectual colectiva para las comunidades indígenas es que hay confusión con respecto a quién o a quiénes debe quedar la titularidad de las marcas de origen. $\mathrm{Al}$ no resolver esta confusión, los derechos quedan en nombre de empresarios que no hacen parte de la comunidad, que no conocen su cultura ni la simbología tan profunda que expresan los tejidos, explica Jacanamijoy ${ }^{42}$.

Por tanto, el principal elemento que se debe considerar en la orientación de esquemas de propiedad colectiva para comunidades indígenas es superar la noción

38 Ojeda, G. Plan especial de salvaguardia del sistema normativo Wayuu, aplicado por el palabrero, Maicao, Guajira, Junta Mayor de palabreros, 2013.

39 Polo. Op, cit., p. 12.

40 Barrera, G.; Quiñonez, A. y Jacanamijoy, J. Op. cit.

41 Ibidem, p. 45.

42 Jacanamijoy, J. Documento base para la implementación de planes, programas y proyectos hacia la autonomía artesanal en el pueblo indígena Kamsá del municipio de Sibundoy, Putumayo, Colombia, Sibundoy, Putumayo, documento de trabajo, 2012. 
LA NECESIDAD DE FORTALECER LA PROTECCIÓN DE LA PROPIEDAD INTELECTUAL EN LOS

TEJIDOS WAYÚU DEBIDO A LAS NUEVAS DINÁMICAS DE PRODUCCIÓN EN LA COMUNIDAD

según la cual el conocimiento indígena es de dominio público, como se encuentra consignado en la Ley 23 de 1982, pues es algo que ha venido afectando sus derechos sobre su propiedad, incluso sobre su legado y su cultura, en términos de Polo y Ojeda ${ }^{43}$.

En particular esta situación ha generado inestabilidades determinantes en la trascendencia cultural y en la sostenibilidad de las comunidades indígenas. Sin embargo, el problema va más allá del plano cultural y se inserta también en situaciones de tipo económico:

Igualmente, se están propiciando dependencias económicas, pues los pueblos deben pagar tarifas establecidas para la continuidad de su hacer creativo con compromisos económicos que nunca habían tenido, entonces surge la inquietud acerca de su permanencia si no tienen los recursos para pagarlas ${ }^{44}$.

Si bien en Colombia el tema sobre la protección de los conocimientos tradicionales ha venido siendo una preocupación constante, lo cual se evidencia en el desarrollo de estudios y consultorías orientadas a conocer los conocimientos tradicionales, a entender sus relaciones particulares con el territorio y con la expresión de un saber comunitario, la realidad es que en el país no existen las condiciones adecuadas para orientar elementos formales de política pública y legislación asociada ${ }^{45}$. Como ejemplo de una de esas consultorías, se resalta la participación de Colombia en el Comité Intergubernamental sobre Propiedad Intelectual, Recursos Genéticos, Conocimientos Tradicionales y Folclore, en el año 2007, en el cual se ha reconocido la necesidad de establecer un sistema de protección sui generis para todo lo que tiene que ver con los conocimientos tradicionales en el país:

Dada la naturaleza particular de los recursos que abarcan los conocimientos tradicionales, se justifica la existencia de un Sistema de Protección Sui-Generis, que debe reconocer las particularidades de los casos en que los procesos de acceso afecten la dimensión del conocimiento tradicional, y en función de lo anterior, regular las prácticas asociadas con el fin de asegurar tanto la protección de los conocimientos mismos, como la distribución justa y equitativa de los beneficios ${ }^{46}$.

Sin embargo, a pesar de estos reconocimientos, en el país se evidencian limitaciones en la regulación para proteger los derechos de las comunidades indígenas en lo que se refiere a la propiedad intelectual y a los conocimientos tradicionales, debido a que estas comunidades no tienen el acceso a información pertinente, y porque las

43 Polo, N. y Ojeda, G. Op. cit.

44 Barrera, G.; Quiñonez, A. y Jacanamijoy, J. Op. cit., p. 47.

45 Muñoz, S., Giraldo, D. y Socorro, A. "Mecanismos de protección de los conocimientos tradicionales: el caso de Colombia", en Revista Derecho del Estado, 22, pp. 45-77.

46 República de Colombia. Comentarios del Gobierno de Colombia frente a las cuestiones de conocimientos tradicionales y expresiones culturales tradicionales / expresiones del folclore, 2007. 
iniciativas que existen no se han logrado articular en instancias legítimas de participación y concertación. Tampoco se ha promovido el desarrollo de estrategias que respondan a las necesidades de las comunidades, a través de procesos participativos que promuevan la sincronización y la colaboración entre las autoridades indígenas, el Estado y las comunidades.

Se evidencia, por tanto, que no existe una normativa específica para la protección de los conocimientos tradicionales en Colombia, razón por la cual los mecanismos de protección de la propiedad intelectual de las comunidades indígenas se han venido desarrollando a través de un conjunto de normas dispersas, en las cuales se consideran de manera aislada diferentes elementos que deberían estar integrados en un solo cuerpo normativo. Dentro de dichos elementos se destaca, por ejemplo, aquellos asociados con la autonomía y organización de los resguardos indígenas, con la protección del territorio, el reconocimiento del derecho a la consulta y la distribución equitativa de beneficios ${ }^{47}$.

En relación concreta con el tema de la propiedad intelectual y el conocimiento tradicional, el país se rige de acuerdo con lo que se encuentra dispuesto la Decisión 486 de 2000 de la Comunidad Andina (CAN). En particular, sobre este documento normativo se destaca el artículo No. 3, referente al patrimonio Biológico y Genético los Conocimientos Tradicionales:

Los Países Miembros asegurarán que la protección conferida a los elementos de la propiedad industrial se concederá salvaguardando y respetando su patrimonio biológico y genético, así como los conocimientos tradicionales de sus comunidades indígenas, afroamericanas o locales. En tal virtud, la concesión de patentes que versen sobre invenciones desarrolladas a partir de material obtenido de dicho patrimonio o dichos conocimientos estará supeditada a que ese material haya sido adquirido de conformidad con el ordenamiento jurídico internacional, comunitario y nacional ${ }^{48}$.

Por otro lado, en cuanto al registro y comercialización de las marcas, en el artículo n. ${ }^{\circ} 136-\mathrm{g}$ ) de la Decisión se establece que en ningún caso se pueden registrar signos que incluyan el nombre de alguna comunidad indígena o que hagan parte de su expresión cultural, a no ser que la comunidad haya expresado su aprobación al respecto. Sin embargo, se evidencia que en la realidad la aplicación de esta norma no se efectúa de manera adecuada, pues son constantes las vulneraciones de los derechos de propiedad intelectual de las comunidades indígenas en los procesos de duplicación y comercialización de sus productos, incluso cuando no se cuenta con ningún tipo de autorización por parte de las comunidades. 
LA NECESIDAD DE FORTALECER LA PROTECCIÓN DE LA PROPIEDAD INTELECTUAL EN LOS TEJIDOS WAYÚU DEBIDO A LAS NUEVAS DINÁMICAS DE PRODUCCIÓN EN LA COMUNIDAD

Esto se debe, explica Tobón ${ }^{49}$, a que las tendencias proteccionistas sobre los derechos de las comunidades indígenas y sus conocimientos tradicionales contrastan fuertemente con las decisiones que se establecen en el país en lo relacionado con acuerdos comerciales, en los cuales se pasa por alto la protección de las figuras de propiedad intelectual sobre los conocimientos tradicionales.

Estas violaciones frecuentes a los derechos de las comunidades indígenas se generan también debido a las consideraciones incluidas en la ley 23 de 1982, cuando se dispone, según el artículo 189, que "El arte indígena, en todas sus manifestaciones, inclusive, danzas, canto, artesanías, dibujos y esculturas pertenece al patrimonio cultural". Por otro lado, en el artículo 187 de la misma ley se plantea que las obras folclóricas y tradicionales de autores desconocidos pertenecen al dominio público. El problema es que el carácter colectivo de las obras indígenas hace que en la mayoría de los casos sea difícil identificar a los autores, por lo cual se consideran como anónimas y, por tanto, correspondientes al dominio público.

Ante estas problemáticas, además de separar los conocimientos y prácticas indígenas de los elementos que componen al dominio público, resulta esencial favorecer la protección de la propiedad intelectual colectiva a partir de un reconocimiento del artículo 246 de la Constitución Política de Colombia, mediante el cual se establece que los pueblos indígenas pueden ejercer funciones jurisdiccionales dentro de su ámbito territorial, de conformidad con sus propias normas y procedimientos, siempre que no sean contrarios a la Constitución y leyes de la República. En este sentido, como lo indican Caviedes y Fuentes ${ }^{50}$, es preciso generar formas de coordinación de la jurisdicción especial de las comunidades indígenas con el sistema judicial nacional en lo que se refiere a la propiedad intelectual.

Es importante que se establezca el desarrollo de una coordinación jurisdiccional entre el Estado y las comunidades indígenas, para que a través de un trabajo conjunto y participativo sea posible definir las formas y mecanismos por medio de los cuales se garanticen los derechos de propiedad intelectual, a través de un conjunto de normas que sean coherentes con la cosmovisión de las comunidades y con lo que representa para el pueblo la producción artesanal de las mochilas en tanto elemento cultural y patrimonial de la comunidad, de la nación y de la humanidad. Lo anterior exige propiciar espacios de participación con la comunidad para conocer sus necesidades y requerimientos especiales en temas de propiedad intelectual.

La propuesta, por tanto, implica orientar el diseño de un sistema de protección local que parta de una comprensión detallada de la idiosincrasia de cada comunidad con la finalidad de ir acumulando experiencias y conocimientos que permitan promover el desarrollo de un marco legal ajustado a las particularidades de las co-

49 Тово́n, N. "Un enfoque diferente para la protección de los conocimientos tradicionales de los pueblos indígenas", en Estud. Socio-Juríd., Bogotá, 9(1), pp. 96-129.

50 Caviedes. C. y Fuentes. H. (2011). "Acuerdos de propiedad intelectual: ¿Nos acercan al desarrollo?”, en Apuntes del CENES, 30(51), pp. 141-164. 
munidades y evitando de esta manera el desarrollo de normas que seguirían siendo demasiado generales y que terminan irrespetando los derechos de las comunidades.

Una regulación de este tipo ayudaría a especificar el desarrollo de medidas penales para prevenir la apropiación, uso y comercialización indebida de las obras que expresan el desarrollo de conocimientos tradicionales indígenas. También es preciso avanzar en lo que tiene que ver con la posibilidad de probar legalmente que hay una aprobación de la comunidad que entrega el conocimiento o el producto tradicional. Finalmente, es preciso demostrar la repartición equitativa de los beneficios, de tal forma que la comercialización d ellos productos indígenas genere regalías para la comunidad que sean equivalentes con los beneficios que obtiene, por ejemplo, los exportadores.

Se propone así partir del conocimiento de las leyes de origen de los creadores de los tejidos, conocer a fondo sus experiencias asociadas a los procesos de creación, además de las transformaciones que han tenido que afrontar y de los nuevos medios de obtención de la materia prima.

También es preciso legalizar procesos de apropiación consentida para la comercialización de las creaciones artesanales, todo a través de un proceso en el cual se pueda fortalecer la autonomía comunitaria de los pueblos indígenas, su capacidad de organización y asociación como eje fundamental desde el cual se puede garantizar la protección de sus derechos.

Es importante que en Colombia se establezca un proceso mediante el cual las leyes de propiedad intelectual se actualicen de acuerdo con los requerimientos de las comunidades y con sus medios particulares de expresión ${ }^{51}$. Además, se debe considerar el enfoque especial que se le ha dado a la investigación y análisis sobre el tema de derechos de autor, a través de la conformación de mecanismos, estamentos y comisiones centradas en analizar los temas asociados a la propiedad intelectual, en la creación colectiva ${ }^{52}$.

\section{CONCLUSIONES}

A través de los últimos años y hasta la actualidad, la comunidad wayúu ha tenido que enfrentar una serie de compromisos económicos, comerciales y productivos que antes no tenía, debido a las nuevas dinámicas comerciales asociadas a la venta de sus tejidos y la entrada en el panorama de empresas que se han dedico a comercializar los productos en el exterior.

Lo anterior ha generado una trasformación progresiva de los procesos de producción, en medio de un cada vez más notable desconocimiento de los derechos de autor $y$ de la propiedad intelectual que debe tener la comunidad sobre sus productos.

51 Ramírez, Z. Tejidos Wayúu: Alta y Media Guajira. Bogotá: Artesanias de Colombia, 1998.

52 Contreras, J. "El aparato teórico en la estructura tradicional del sistema de propiedad intelectual”, en Vniversitas, 135, 2017, pp. 99-130. 
Estas situaciones permiten observar que la protección de los conocimientos tradicionales no puede reducirse a la adopción de figuras tradicionales de propiedad intelectual, ya que dichos conocimientos integran la cultura y los procesos vitales de las comunidades, razón por la cual es preciso considerar los procesos colectivos a los cuales se integra la producción de creación de formas artísticas que expresan una relación particular con el territorio. Hasta ahora, la limitada reglamentación que existe en Colombia sobre la protección de los conocimientos tradicionales, como la Decisión 486 de 2000 de la Comunidad Andina y la Ley 23 de 1982, no ha permitido entender en el país que el tema de los conocimientos tradicionales es complejo, pues se involucran en su desarrollo una serie de elementos que resultan claves en la vida, desarrollo y expresión cultural de las comunidades.

Por otro lado, no se cuenta con un instrumento normativo específico desde el cual se otorgue y garantice la protección a las comunidades indígenas, desde una concepción que integre la comprensión detallada sobre su cosmovisión ${ }^{53}$. Esta situación obliga a formular el desarrollo de un marco normativo que pueda sintetizar dos líneas específicas de protección: una en la cual la propiedad intelectual se garantice de acuerdo con una comprensión la realidad económica actual y las necesidades del mercado; y otra en la cual se comprenda la importancia de los conocimientos tradicionales como elementos vitales para la expresión cultural y supervivencia de las comunidades indígenas.

En síntesis, se puede observar que los limitados esfuerzos que hasta ahora se han desarrollado en Colombia para proteger los conocimientos tradicionales de las comunidades indígenas han fallado por las siguientes razones. En primer lugar, porque los creadores de los conocimientos tradicionales no comparten las nociones de la propiedad privada e individualista en la cual se basa generalmente la aplicación de los derechos de propiedad intelectual. En segundo lugar, porque en Colombia no existe una clasificación de los conocimientos tradicionales que ayude a orientar un marco legal pertinente, y porque se destaca la ausencia de espacios de diálogo y participación con las comunidades, a efectos de conocer, por ejemplo, cuánto deben durar los derechos, cómo se deben proteger y cuáles deben ser las sanciones impuestas a aquellos que violen los derechos. Finalmente, se destacan dificultades para definir figuras comunes en el Derecho pero que no son comprensibles ni asimilables para las comunidades indígenas, como "consentimiento previo informado" y "repartición equitativa de beneficios".

En particular, se reconoce que la necesidad de promover esquemas efectivos de propiedad intelectual colectiva que partan de una comprensión integral sobre todo el proceso de producción de los tejidos, sus sentidos y sus significados, es clave no solo para garantizar la protección de los derechos de la comunidad wayúu sobre sus obras, sino también para asegurar su permanencia, su subsistencia y sus identidades culturales. 
Esto implica dejar de considerar a las creaciones y conocimientos indígenas como elementos asociados al dominio público, y establecer de esta manera medidas concretas de propiedad intelectual que surjan del desarrollo de espacios participativos, a partir de los cuales sea posible comprender los puntos de vista, las problemáticas y las necesidades de la comunidad.

\section{REFERENCIAS}

ACEA, Y. "El sistema de propiedad intelectual y la protección de los conocimientos tradicionales. Apuntes necesarios", en Alegatos, 12(3), 2016, pp. 25-55.

Aguilar, I., \& Márquez, P. El arte del tejido entre los hombres de la etnia Wayuu de La Guajira colombo-venezolana. Colombia: Fundación Universidad de América. 2016.

Alarcón, J. "The Wayuu society, between chimera and reality", en Gazeta de Antropología, 2006, 22, artículo 21.

Apshana, V. En: Harker, S. Wayuu peuple du désert colombien. Bogotá, Villegas Editores. 1998.

Artesanías de Colombia. Memoria de oficio tejeduría del pueblo indígena wayúu. [En línea]. 2014. Disponible en:

Barrera, G., QuiÑonez, A., y Jacanamijoy, J. "Riesgos y tensiones de las marcas colectivas y denominaciones de origen de las creaciones colectivas artesanales indígenas", en Apuntes, 2009, 27(1), pp. 36-51.

Benavidess, G. y Forero, P. "Crecimiento endógeno: conocimiento y patentes", en Revista de Economía Institucional, 4(6), 2002, pp. 109-131.

Castilleja, A. "Patrimonio cultural; ¿¿de quién y para qué?", en $C N D H$, Argumentos para la Defensa y Protección del Patrimonio Cultural de Pueblos y Comunidades Indigenas y Afrodescendientes en México y América Latina, México, CNDH, 2019, pp. 83-87.

Caviedes. C. y Fuentes. H. "Acuerdos de propiedad intelectual: ¿Nos acercan al desarrollo?”, en Apuntes del CENES, 30(51), 2011, pp. 141-164.

CHÁvez, C. Cultura y globalización: la seguridad societal de la comunidad indígena wayú y la comercialización de los tejidos tradicionales, Universidad Colegio Mayor de Nuestra Señora del Rosario, 2017.

Chen, Y. \& T. "Puttitanun Intellectual property rights and innovation in developing countries", en Journal of Development Economics, 78(2), 474-493. 2005.

Comunidad Andina (CAN). Decisión 486 de 2000.

Comunidad Wayúu. Plan salvaguarda del pueblo Wayúu. [En línea]. 2014. Disponible en: https://siic.mininterior.gov.co/sites/default/files/pueblo_wayuu_norte_albania_-_diagnostico_comunitario.pdf

Contreras, J. "El aparato teórico en la estructura tradicional del sistema de propiedad intelectual”, en Vniversitas, 135. 2017, pp. 99-130. 
LA NECESIDAD DE FORTALECER LA PROTECCIÓN DE LA PROPIEDAD INTELECTUAL EN LOS TEJIDOS WAYÚU DEBIDO A LAS NUEVAS DINÁMICAS DE PRODUCCIÓN EN LA COMUNIDAD

DíAz, A. América Latina y el Caribe: la propiedad intelectual después de los tratados de libre comercio, CEPAL, 2008, Santiago de Chile.

Guerra, W. "Los conflictos interfamiliares Wayuu", en Fronesis, 13(1), 2006, pp. 40-56.

Hostein, N. "El pueblo wayúu de La Guajira colombo-venezolana: un panorama de su Cultura", en Cuadernos de Antropología, 20, 2010.

http://www.wipo.int/edocs/pubdocs/es/intproperty/450/wipo_pub_450.pdf

https://www.artesaniasdecolombia.com.co/PortalAC/C_noticias/la-mochila-wayuparte-de-la-tradicion-de-colombia_5070

JaCanamijoy, J. Documento base para la implementación de planes, programas y proyectos hacia la autonomía artesanal en el pueblo indígena Kamsá del municipio de Sibundoy, Putumayo, Colombia. Sibundoy, Putumayo, documento de trabajo, 2012.

López, A. La experiencia de Panamá respecto a la protección de las artesanías y su relación con la propiedad intelectual. Disponible en: http://www.impi.gob.mx/ work/sites/IMPI/resources/LocalContent/2383/1/ARESIO.pdf (20 de septiembre del 2011).

Manquillo, N. 2. "La vulnerabilidad del sector artesanal colombiano en el ámbito nacional e internacional como 'negocio de conocimiento tradicional. Estudio de caso: la denominación de origen artesanal 'Guacamayas' en el Acuerdo Comercial entre Colombia, Perú y la Unión E”, en Revista La Propiedad Inmaterial. 28 (dic. 2019), pp. 167-209.

MejíA, P. Situación sociolingüistica del Wayuunaiki. (Tesis de maestría). Universidad Nacional de Colombia, Bogotá, Colombia. 2011.

Muñoz, S., Giraldo, D., y Socorro, A. "Mecanismos de protección de los conocimientos tradicionales: el caso de Colombia", en Revista Derecho del Estado, 22, pp. 45-77.

Ojeda, G. Plan especial de salvaguardia del sistema normativo Wayuu, aplicado por el palabrero. Maicao, Guajira, Junta Mayor de palabreros, 2013.

Organización Mundial de la Propiedad Intelectual. [online] ¿Qué es la Propiedad Intelectual?. 2017. Obtenido de:

Pérez, J. "La artesanía en el Derecho", en Vniversitas 125, 2012, pp. 287-318.

Polo, N. y OjedA, G. "Mirada a la cultura Wayuu, base de su sistema normativo", en Verbum, 9(9), 2014, pp. 109-117.

Polo, N. "A gaze to Wayu's Culture, Base of his Normative System", en Paripex -Indian Journal of Research, 4(250), 2015, pp. 72-75.

Polo, N. "La cultura y el sistema de normas Wayuu", en Nuevo Derecho, 11(17), 2015, pp. 65-78.

Ramírez, Z. Tejidos Wayúu: Alta y Media Guajira, Bogotá, Artesanías de Colombia, 1998.

Rangel, H. La observancia de los derechos de propiedad intelectual, Organización Mundial de la propiedad Intelectual, 2015. 
República de Colombia. Comentarios del Gobierno de Colombia frente a las cuestiones de conocimientos tradicionales y expresiones culturales tradicionales / expresiones del folclore, 2007.

República de Colombia. Constitución Política, 1991.

Rubio, A et al. Guía de la artesanía y el derecho de autor. Coautoría de Artesanías de Colombia y la Dirección de Derecho de Autor, 2010.

SAler, B. "Los wayú (guajiro)", en Los aborígenes de Venezuela, Caracas, Fundación La Salle y Monte Ávila, 1987.

SÁnchez, B. "Espiritualidad Wayuu en relación con la muerte", en Revista de Literatura Hispanoamericana. 2016. 2(4), pp. 22-54.

SÁncheZ, B. "Una aproximación al arte wayúu en; el lenguaje simbólico del mito", en Revista de Literatura.

Sempere, A. Propiedad intelectual conceptos básicos, España, Universidad de Alicante. 2015.

Silberleib, L. "El derecho, la propiedad intelectual y el entorno digital", en Información, Cultura Y Sociedad, 1, (4), 2011, pp. 34-66.

Tejedor, M.; Gil, F. y Tejedor, P. "Derechos de propiedad intelectual (dpi) y crecimiento económico: una revisión”, en Rev. Fac. Cienc. Econ, 16(1), 2016, pp. 225-243.

ТовóN, N. "Un enfoque diferente para la protección de los conocimientos tradicionales de los pueblos indígenas", en Estud. Socio-Juríd., Bogotá (Colombia), 9(1), pp. 96-129

URIBE, M. La transformación de la Propiedad Intelectual. Ediciones Doctrina y Ley, 2005, Bogotá D.C.

VArgas, S. y Lascarro, A. "Derecho a la autodeterminación de los pueblos indígenas en el ordenamiento jurídico colombiano”, en Revista de Estudios Sociales, 2015. (53), pp. 65-76.

Zerda, A. y Forero, D. Los derechos de propiedad intelectual sobre los conocimientos de las comunidades étnicas, 2009. 